\title{
WEAKLY ADDITIVE COHOMOLOGY
}

\author{
E. SPANIER
}

\begin{abstract}
In this paper the concept of weakly additive cohomology theory is introduced as a variant of the known concept of additive cohomology theory. It is shown that for a closed $A$ in $X$ the singuisr homology of the pasi $(X, X-A)$ (with some fixed coefficient gropu) regarded as a furcter of $A$ is a weakly additive cohomology theory on any collectionwise normal space $X$. Furthermore, every compactly supported cohomology theory is weakly additive.

The main result is a comparison theorem for two cohomology theories on $X$ both of which are additive or both of which are weakly additive which ercomposses the previously known compauson theorems.
\end{abstract}

A cohomology theory $H, \delta$ on a topological space (all spaces will be assumed to be normal Hausdorf spaces) consists of a continuous contravariant functor $H$ from the category of closed subsets of $X$ (and inclusion maps between them) to the category of graded abelian groups (and homomorphisms of degree 0 between them) and for every two closed sets $A, B$ in $X$ a natural transformation $\delta: H(A \cap B) \rightarrow H(A \cup B)$ of degree 1 such that $M V$ exactness is satisfied. For relevant definitions and more details see $[3,4,5]$.

For any space $X$ there is a cohomology theory ${ }^{\Delta} H, \Delta \delta$ on $X$ in which ${ }^{\Delta} H^{q}(A)=$ $H_{-q}(X, X-A)$ (relative singular homology with some fixed coefficient group) and $\Delta_{\delta}$ is the connecting homomorphism

$$
\triangle_{\delta}: H_{-q}(X, X-(A \cap B)) \longrightarrow H_{-q-1}(X, X-(A \cup B))
$$

of the exact relative Mayer-Vietoris homology sequence of $(X, X-A)$ and $(X, X-B)$. In case $X$ is locally compact, this cohomology theory has compact supports (Example (3.3) in [5]). However, more generally on any collectionwise normal space (in particular, any paracompact space) it satisfies the property of weak additivity which we proceed to define.

The author gratefully acknowledges partial support by the Centre de Recerca Matematica. of the Institute d'Estudis Catalans of the Universitat Autònoma de Barcelona in Bellaterra (Barcelona); Spain during the preparation of this paper. 
If $\left\{A_{j}\right\}_{j \in J}$ is a discrete family (i.e. every point of $X$ has a neighborhood which meets at most one member of the family) of closed subsets of $X$ and $H, \delta$ is a cohomology theory on $X$, there is a homomorphism $\sigma: H\left(\cup_{j \in J} A_{j}\right) \rightarrow$ $\Pi_{j \in J} H\left(A_{j}\right)$ defined by $\sigma(u)=\left\{u \mid A_{j}\right\}_{j \in J}$ for $u \in H\left(\cup_{j \in J} A_{j}\right)$. The homology theory is additive $[1,3]$ if $\sigma$ is an isomorphism for all discrete families $\left\{A_{j}\right\}$ in $X$. We define a homomorphism $\imath: \oplus_{j \in J} H\left(A_{j}\right) \rightarrow H\left(\cup_{j \in J} A_{j}\right)$ which will be needed below to define the concept of weak additivity.

Note that MV exactness implies that if $A, B$ are disjoint closed sets in $X$ then for any cohomology theory $H, \delta$ on $X$ there is an isomorphism $\alpha: H(A \cup B) \approx$ $H(A) \oplus H(B)$ where $\alpha(u)=(u|A, u| B)$ for $u \in H(A \cup B)$. Straightforward induction shows that for a finite collection $\left\{A_{i}\right\}_{i=1}^{n}$ of pairwise disjoint closed sets there is an isomorphism

$$
\oplus_{i=1}^{n} H\left(A_{i}\right) \approx H\left(\cup_{i=1}^{n} A_{i}\right) .
$$

If $\left\{A_{j}\right\}_{j \in J}$ is a discrete family of closed subsets of $X$ and $J^{t} \subset J$ there is an isomorphism

$$
\alpha_{J^{\prime}} ; H\left(\cup_{j \in J} A_{j}\right) \approx H\left(\cup_{j \in J^{\prime}} A_{j}\right) \oplus H\left(\cup_{j \notin J^{\prime}} A_{j}\right)
$$

In particular, if $F \subset J$ is any finite set there is a monomorphism $i_{F}: \oplus_{j \in F} H\left(A_{j}\right) \rightarrow H\left(\cup_{j \in J} A_{j}\right)$ equal to the composite

$\oplus_{j \in F} H\left(A_{j}\right) \approx H\left(\cup_{j \in F} A_{j}\right) \subset H\left(\cup_{j \in F} A_{j}\right) \oplus H\left(\cup_{j \notin F} A_{j}\right) \stackrel{\alpha_{F}^{-3}}{\approx} H\left(\cup_{j \in J} A_{j}\right)$. In case $F, F^{\prime}$ are finite subsets of $J$ with $F \subset F^{\prime}$ there is a commutative triangle

$$
\begin{gathered}
\oplus_{j \in F} H\left(A_{j}\right) \subset \oplus_{j \in F^{\prime}} H\left(A_{j}\right) \\
i_{F} \searrow H\left(\cup_{j \in J} A_{j}\right) \\
\swarrow i_{F^{\prime}}
\end{gathered}
$$

Therefore, there is a monomorphism $\underset{\lim }{\longrightarrow}\left\{\oplus_{j \in F} H\left(A_{j}\right) \mid F\right.$ finite $\rightarrow$ $H\left(\cup_{j \in J} A_{j}\right)$. Since $\oplus_{j \in J} H\left(A_{j}\right) \approx \underset{\longrightarrow}{\lim }\left\{\oplus_{j \in F} H\left(A_{j}\right) \mid F\right.$ finite $\}$ we obtain a monomorphism

$$
\iota: \oplus_{j \in J} H\left(A_{j}\right) \longrightarrow H\left(\cup_{j \in J} A_{j}\right) .
$$

$H, \delta$ is said to be weakly additive if $\iota$ is an isomorphism for all discrete families $\left\{A_{j}\right\}$ in $X$.

Example. Let $X$ be a collectionwise normal space and let $\left\{A_{j}\right\}_{j \in J}$ be a discrete family of closed subsets of $X$. Then there is a discrete family of open sets $\left\{U_{j}\right\}_{j \in J}$ such that $A_{j} \subset U_{j}$ for each $j$ (this is the meaning of collectionwise normal space). Then $X=\cup_{j \in J} U_{j} \cup\left(X-\cup_{j \in J} A_{j}\right)$ where $\cup_{j \in J} U_{j}$ and $X-$ $\cup_{j \in J} A_{j}$ are each open in $X$. Therefore, there is an excision isomorphism

$$
H_{-q}\left(\cup_{j \in J} U_{j},\left(\cup_{j \in J} U_{j}\right) \cap\left(X-\cup_{j \in J} A_{j}\right)\right) \approx H_{-q}\left(X, X-\cup_{j \in J} A_{j}\right)={ }^{\triangle} H^{q}\left(\cup_{j \in J} A_{j}\right) .
$$


Since $\left(U_{j \in J} A_{j}\right) \cap U_{j}=A_{j},\left(\cup_{j \in J} U_{j}\right) \cap\left(X-U_{j \in J} A_{j}\right)=U_{j \in J}\left(U_{j}-A_{j}\right)$ so that

$$
\begin{gathered}
H_{-q}\left(\cup_{j \in J} U_{j},\left(\cup_{j \in J} U_{j}\right) \cap\left(X-\cup_{j \in J} A_{j}\right)\right)=H_{-q}\left(U_{j \in J} U_{j}, \cup_{j \in J}\left(U_{j}-A_{j}\right)\right) \\
\approx \oplus_{j \in J} H_{-q}\left(U_{j}, U_{j}-A_{j}\right) \approx \oplus_{j \in J} H_{-q}\left(X, X-A_{j}\right)=\oplus_{j \in J} H^{q}\left(A_{j}\right) .
\end{gathered}
$$

It follows that $\imath: \oplus_{j \in J}{ }^{\Delta} H^{q}\left(A_{j}\right) \rightarrow \Delta^{q} H^{q}\left(\cup_{j \in J} A_{j}\right)$ is an isomorphism so $\Delta_{H}, \Delta_{\delta}$ is a weakly additive cohomology theory on every collectionwise normal space.

There is the following characterization of weak additivity.

Lemma. Let $H, \delta$ be a cohomology theory on $X$ and suppose for every $u \in$ $H\left(\cup_{j \in J} A_{j}\right)$, where $\left\{A_{j}\right\}$ is a discrete family of closed subsets of $X$, there is some finite subset $F \subset J$ such that $u \mid \cup_{j \notin F} A_{j}=0$. Then $H, \delta$ is weakly additive.

Proof: The hypothesis implies that $u$ in the image of $i_{F}: \oplus_{j \in F} H\left(A_{j}\right) \rightarrow$ $H\left(\cup_{j \in J} A_{j}\right)$. This implies that the homomorphism $\iota: \oplus_{j \in J} H\left(A_{j}\right) \rightarrow$ $H\left(\cup_{j \in J} A_{j}\right)$ is an epimorphism. Since $\iota$ is always a monomorphism, it is an isomorphism and so $H, \delta$ is weakly additive.

Corollary. Every compactly supported cohomology theory is weakly additive.

Proof: If $\left\{A_{j}\right\}_{j \in J}$ is a discrete family of closed subsets of $X$ and $C \subset \cup_{j \in J} A_{j}$ is a compact subset, there is some finite set $F \subset J$ such that $C \subset \cup_{j \in F} A_{j}$. Suppose $H, \delta$ is a compactly supported cohomology theory on $X$ and $u \in$ $H\left(\cup_{j \in J} A_{j}\right)$ where $\left\{A_{j}\right\}$ is a discrete family of closed sets $\operatorname{in} X$. Then $\cup_{j \in J} A_{j}=$ $B \cup C$ where $B$ is closed and $C$ is compact and $u \mid B=0$. Let $F \subset J$ be a finite subset such that $C \subset \cup_{j \in F} A_{j}$. Then $\cup_{j \notin F} A_{j} \subset B$ so $u \mid \cup_{j \notin F} A_{j}=0$.

By the preceding Lemma, $H, \delta$ is weakly additive.

The comparison theorems previously established $[1,3,4,5]$ have been valid for compactly supported or additive cohomology theories. The above corollary asserts that compactly supported cohomology theories are weakly additive, and the following comparison theorem, which is the main result of the paper, is a way of generalizing and of unifying the two comparison theorems into basically one.

Theorem, Let $\varphi: H, \delta \rightarrow H^{\prime}, \delta^{\prime}$ be a homomorphism between cohomology theories both of which are weakly additive (or both additive) and having paracompact supports on the same space $X$. Suppose there is n such that $\varphi_{x}: H(x) \rightarrow$ $H\left(x^{\prime}\right)$ is an n-equivalence for all $x \in X$. If $H, \delta$ and $H^{\prime}, \delta^{\prime}$ are both nonnegative or if $X$ is locally finite dimensional, then $\varphi_{A}: H(A) \rightarrow H^{\prime}(A)$ is an $n$-equivalence for all closed $A \subset X$. 
Proof: It follows from Theorem (2.5) of [4] that it suffices to prove the theorem for closed paracompact subsets of $X$. We need the following result (Theorem (5.5) of $[2])$.

Let $X$ be a paracompact space and $C$ a collection of closed subsets of $X$ such that:

1) $B \in \mathcal{C}$ and $B^{\prime}$ closed in $B \Rightarrow B^{\prime} \in \mathcal{C}$.

2) Every $x \in X$ has a closed neighborhood $N \in \mathcal{C}$.

3) $B_{1}, B_{2} \in \mathcal{C}$ and $B_{1} \cup B_{2}=$ int $_{B_{1} \cup B_{2}} B_{1} \cup$ int $_{B_{1} \cup B_{2}} B_{2} \Rightarrow B_{1} \cup B_{2} \in \mathcal{C}$.

4) If $\left\{B_{j}\right\}_{j \in \mathcal{C}}$ is a discrete family of elements of $\mathcal{C}$, then $\cup_{j \in J} B_{j} \in \mathcal{C}$. Then $X \in \mathcal{C}$.

In the proof we shall have occasion to use this theorem several times. In each case it will follow from the definition of $\mathcal{C}$ that $\mathcal{C}$ has property 1). From the fact that $\varphi_{x}: H(x) \rightarrow H^{\prime}(x)$ is an $n$-equivalence for all $x \in X$ and the continuity of $H$ and $H^{\prime}$ it will follow that $\mathcal{C}$ has property 2). Because both $H, \delta$ and $H^{\prime}, \delta^{\prime}$ are weakly additive or both are additive it will follow that $\mathcal{C}$ has property 4 ). It is only property 3) that will require some attention and in each case the appropriate diagram will be presented.

Corresponding to the two hypotheses of nonnegativity or finite dimensionality we distinguish two cases in the proof.

The proof is analogous to that of Theorem 3.3 of [1].

Case 1. If both $H, \delta$ and $H^{\prime}, \delta^{\prime}$ are nonnegative we prove $\varphi_{A}: H^{q}(A) \rightarrow$ $H^{\prime q}(A)$ is an isomorphism for all $q<n$ for all closed paracompact $A \subset X$ by induction on $q$. By nonnegativity this is true for $q=0$. Assume $0 \leq q \leq n$ and $\varphi_{A}$ is an isomorphism in dimension $q-1$ for all such $A$. We prove $\varphi_{A}: H^{q}(A) \rightarrow$ $H^{\prime q}(A)$ is a monomorphism. Let $u \in H^{q}(A)$ be a fixed element in $\operatorname{ker} \varphi_{A}$ and let $\mathcal{C}$ be the collection of all closed sets $B \subset A$ such that $u \mid B=0$. As remarked above $\mathcal{C}$ has properties 1),2), 4). If $B_{1}, B_{2} \in \mathcal{C}$ there is a diagram with exact rows in which each square commutes or anticommutes

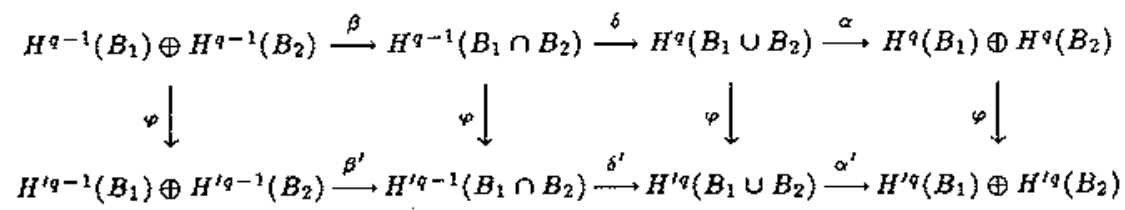

By the inductive hypothesis both of the first two left hand vertical homomorphisms are isomorphisms. It follows by diagram chasing that $B_{1} \cup B_{2} \in \mathcal{C}$ so $\mathcal{C}$ satisfies 3 ). Therefore, $A \in \mathcal{C}$ so $\varphi_{A}: H^{q}(A) \rightarrow H^{r q}(A)$ is a monomorphisrn.

Now assume $q<n$ and $\varphi_{A}$ is a $q$-equivalence for all closed paracompact $A \subset X$. We prove $\varphi_{A}: H^{q}(A) \rightarrow H^{\prime q}(A)$ is an epimorphism. Let $u^{\prime} \in H^{q}(A)$ be fixed and let $\mathcal{C}$ be the collection of all closed $B \subset A$ such that $u^{\prime} \mid B \in$ $\operatorname{im}\left[\varphi_{B}: H^{q}(B) \rightarrow H^{\prime q}(B)\right]$. Then $\mathcal{C}$ has properties 1$)$, 2), 4). If $B_{1}, B_{2} \in \mathcal{C}$ there is a diagram with exact rows in which each square commutes or anticom- 
mutes.

$$
\begin{aligned}
& H^{q-1}\left(B_{1} \cap B_{2}\right) \stackrel{\delta}{\longrightarrow} H^{q}\left(B_{1} \cup B_{2}\right) \stackrel{\alpha}{\longrightarrow} H^{q}\left(B_{1}\right) \oplus H^{q}\left(B_{2}\right) \stackrel{\beta}{\longrightarrow} H^{q}\left(B_{1} \cap B_{2}\right) \\
& \varphi \downarrow \varphi \downarrow \varphi \varphi \downarrow \varphi \\
& H^{\prime q-1}\left(B_{1} \cap B_{2}\right) \stackrel{\delta^{\prime}}{\longrightarrow} H^{\prime q}\left(B_{1} \cup B_{2}\right) \stackrel{\alpha^{\prime}}{\longrightarrow} H^{\prime q}\left(B_{1}\right) \oplus H^{\prime q}\left(B_{2}\right) \stackrel{\beta^{\prime}}{\longrightarrow} H^{\prime q}\left(B_{1} \cap B_{2}\right)
\end{aligned}
$$

By the inductive hypothesis the right hand vertical map is a monomorphism and the left hand vertical map is an isomorphism. It follows by diagram chasing that $B_{1} \cup B_{2} \in \mathcal{C}$ so $\mathcal{C}$ satisfies 3$)$. Therefore, $A \in \mathcal{C}$ so $\varphi_{A}: H^{q}(A) \rightarrow H^{\prime}(A)$ is an epimorphism.

This completes the proof of the Theorem in Case 1.

Case 2. If $X$ is locally finite dimensional (i.e. every point of $X$ has a closed finite dimensional neighborhood) it follows from Proposition (2.9) of [5] (which is clearly also valid if both cohomology theories in its statement are weakly additive instead of additive) that it suffices to prove that $\varphi_{A}: H(A) \rightarrow H^{\prime}(A)$ is an $n$-equivalence for all closed paracompact $A$ of finite dimension.

The proof is analogous to that of Theorem (5.4) in [4]. Note that in Case 1 we prove first that for $q<n$ the map $\varphi_{A}: H^{q}(A) \rightarrow H^{\prime} q(A)$ is a monomorphism and use that to prove it is an epimorphism. In case 2 we prove first that for $q<n$ the map $\varphi_{A}: H^{q}(A) \rightarrow H^{\prime} q(A)$ is an epimorphism and use that to prove that $\varphi_{A}: H^{q+1}(A) \rightarrow H^{\prime q+1}(A)$ is a monomorphism.

We prove $\varphi_{A}: H(A) \rightarrow H^{\prime}(A)$ is an $n$-equivalence for all closed finite dimensional paracompact $A$ by induction on $\operatorname{dim} A$. If $\operatorname{dim} A=-1, A=\emptyset$ and $\varphi_{\theta}$ is an isomorphism. Assume $\operatorname{dim} A=m \geq 0$ and the result is valid for all closed paracompact subsets of dimension $<m$.

We show $\varphi_{A}: H^{q}(A) \rightarrow H^{\prime q}(A)$ is an epimorphism for $q<n$, Let $u^{\prime} \in$ $H^{\prime q}(A)$ and let $\mathcal{C}$ be the collection of all closed subsets $B \subset A$ such that $u^{\prime} \mid B \in \operatorname{im}\left[\varphi_{B}: H^{q}(B) \rightarrow H^{\prime q}(B)\right]$. Then $\mathcal{C}$ satisfies 1$\left.\left.), 2\right), 4\right)$. If $B_{1}, B_{2} \in \mathcal{C}$ and $B_{1} \cup B_{2}=\operatorname{int}_{B_{1} \cup B_{2}} B_{1} \cup \operatorname{int}_{B_{1} \cup B_{2}} B_{2}$, it follows from Lemrna (5.3) of [4] that there exist closed $C_{1}, C_{2}$ with $C_{1} \subset B_{1}, C_{2} \subset B_{2}, B_{1} \cup B_{2}=C_{1} \cup C_{2}$, and $\operatorname{dim}\left(C_{1} \cap C_{2}\right)<m$. The following diagram has exact rows and commutes up to sign

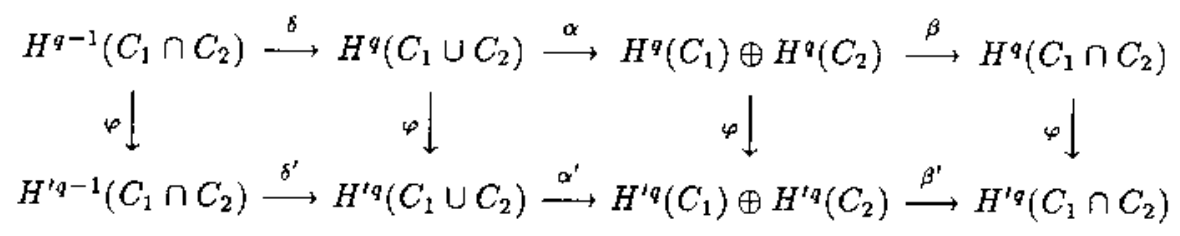

By the inductive hypothesis the two vertical maps at the ends are isomorphisms. By diagram chasing $C_{1} \cup C_{2} \in \mathcal{C}$ so $\mathcal{C}$ satisfies 3 ). Therefore, $A \in \mathcal{C}$ so $\varphi_{A}: H^{q}(A) \rightarrow H^{q}(A)$ is an epimorphism. 
We show $\varphi_{A}: H^{q}(A) \rightarrow H^{\prime q}(A)$ is a monomorphism for $q \leq n$. Let $u \in$ $\operatorname{ker} \varphi_{A}$ and let $C$ be the collection of all closed subsets $B \subset A$ such that $u \mid B=$ 0 . Then $\mathcal{C}$ satisfies 1), 2), 4). If $B_{1}, B_{2} \in \mathcal{C}$ with $B_{1} \cup B_{2}=\operatorname{int}_{B_{1} \cup B_{2}} B_{1} \cup$ int $B_{1} \cup B_{2} B_{2}$ then, as above, there exist closed $C_{1}, C_{2}$ with $C_{1} \subset B_{1}, C_{2} \subset$ $B_{2}, C_{1} \cup C_{2}=B_{1} \cup B_{2}$, and $\operatorname{dim}\left(C_{1} \cap C_{2}\right)<m$. The following diagram has exact rows and commutes up to sign

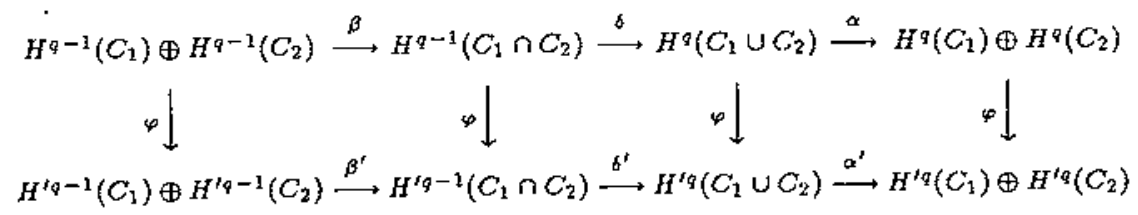

The left hand vertical map is an epimorphism by the frrst part of the pronf, and the second vertical map is an isomorphism by the inductive hypothesis. By diagram chasing it follows that $C_{1} \cup C_{2} \in \mathcal{C}$ so $\mathcal{C}$ satisfies 3 ). Therefore, $A \in \mathcal{C}$ so $\varphi_{A}: H^{q}(A) \rightarrow H^{\prime q}(A)$ is a monomorphism.

This completes the proof in Case 2 and so the Theorem is proved.

\section{References}

1. I.D. LAWSON, Comparison of taut cohomologies, Aeq. Math. 2 (1973), 201-209.

2. E. Michael, Local properties of topological spaces, Duke Math. Jour. 21 (1954), 163-171.

3. E. Spanier, Cohomology isomorphisms, Contemporary Math. 12 (1982), 315-329.

4. E. SPanier, Cohomology with supports, Pac. Jour. of Math. 123 (1986), 447-464.

5. E. SPAnier, Cohomology theories on spaces, Trans. Amer. Math. Soc. 301 (1987), 149-161.

Deptartment of Mathematics

University of California

Berkcley, CA94720

U.S.A.

Rebut el 7 d'Abril de 1989 